\title{
Mischungswärmen und Verbindungsbildung in binären flüssigen Systemen
}

\author{
III. Äther - Chloroform-Systeme * \\ F. BeCKer und M. KIEFER \\ Institut für Physikalische Chemie der Universität Frankfurt a. M. \\ (Z. Naturforsch. 26 a, 1040-1046 [1971] ; eingegangen am 18. März 1971)
}

\begin{abstract}
The enthalpies of mixing at $25^{\circ}$ of diethyl ether, di-n-propyl ether, di-n-butyl ether, di-isopropyl ether, propylene oxide, tetrahydrofuran, and tetrahydropyran with chloroform are determined by an isothermal titration method. As a result, the functions $H^{\mathrm{M}}=f\left(\mathrm{~N}_{\mathrm{CHCl}_{3}}\right)$ are obtained with a step width of 0.025 of the mole fraction and a relative accuracy of 1 per cent or better. Evaluation of the heat of mixing data by means of equilibrium models ("ideal associated mixture") shows that the systems of aliphatic ethers with chloroform behave rather precisely as one-step equilibria of the type $A+B=A B(A=$ ether; $B=$ chloroform). In the systems of cyclic ethers with chloroform, a second equilibrium step, $\mathrm{AB}+\mathrm{B}=\mathrm{AB}_{2}$, must be considered, the importance of which decreases with increasing ring size of the ether. The equilibrium data calculated for the seven ether-chloroform systems are discussed.
\end{abstract}

Nachdem in den beiden vorausgegangenen Mitteilungen ${ }^{1,2}$ gezeigt werden konnte, daß sich der Verlauf der integralen Mischungswärmen in den Systemen Diäthyläther-Chloroform und Tetrahydrofuran-Chloroform in sehr guter Näherung durch ein Einstufen- bzw. Zweistufen-Gleichgewichtsmodell (Modell der „ideal assoziierten Mischung“) wiedergeben läßt, war es von Interesse, vergleichende Untersuchungen über die thermodynamischen Eigenschaften von Mischungen verschiedener aliphatischer und cyclischer Äther mit Chloroform auszuführen. In der vorliegenden Arbeit soll zunächst über Ergebnise an einfachen Äthern berichtet werden. Da eine Anwendung von Gleichgewichtsmodellen auf Mischungskurven nur dann sinnvoll ist, wenn genügend viele und genaue Meßwerte, insbesondere auch bei extremeren Mischungsverhältnissen, vorliegen, haben wir die Mischungswärmen aller untersuchten Systeme mit Hilfe der von uns entwickelten kontinuierlichen Meßmethode ${ }^{3}$ neu gemessen, so daß die Funktionen $H^{\mathrm{M}}=f\left(\mathrm{CHCl}_{3}\right)$ in Form von 40 Funktionswerten mit einer Schrittweite von 0,025 des Molenbruchs und einer Relativgenauigkeit von $1 \%$ und besser zur Verfügung stehen.

Sonderdruckanforderungen an Prof. Dr. F. Becker, Institut für Physikalische Chemie der Universität Frankfurt/M., D-6000 Frankfurt/Main 1, Robert-Mayer-Straße 11.

* Auszugsweise vorgetragen auf der 68. Hauptversammlung der Deutschen Bunsen-Gesellschaft für Physikalische Chemie vom 15.-18. Mai 1969 in Frankfurt a. M.; vgl. Ber. Bunsenges. Phys. Chem. 73, 917 [1969].

\section{Experimentelle Ergebnisse}

Die Messungen wurden mit der in ${ }^{3}$ beschriebenen Apparatur durchgeführt, wo auch das Meßverfahren und die Auswertemethode wiedergegeben sind (vgl. auch ${ }^{4}$ ).

\section{a) Reinigung und physikalische Daten der verwendeten Substanzen}

Chlor of orm: Das Ausgangsprodukt (Merck, DAB 6) enthielt zur Stabilisierung 0,6-1,0\% Äthanol, das durch 7-8-maliges Ausschütteln mit destilliertem Wasser entfernt wurde. Nach Trocknung mit $\mathrm{CaCl}_{2}$ und mit Sikkon (Fluka) wurde das Chloroform unter Reinststickstoff über eine automatische Vigreux-Kolonne mit 10 theoretischen Böden feinfraktioniert, wobei die Destillationsapparatur mit schwarzem Papier umwickelt war, um photochemische Zersetzungen $\mathrm{zu}$ verhindern. Das erhaltene Destillat wurde gekühlt aufbewahrt und innerhalb von $2-3$ Tagen verbraucht.

$$
K_{p 731} 60,0^{\circ} ; n_{\mathrm{D}}^{20,9} 1,4451 ; D_{4}^{25} 1,4799 \text {. }
$$

Äther: Reinste Handelsprodukte der Firmen Fluka bzw. Merck wurden zur Entfernung von Peroxiden zunächst mit konzentrierter wäßriger $\mathrm{FeSO}_{4}{ }^{-}$ Lösung geschüttelt, dann mit 0,5 -proz. $\mathrm{KMnO}_{4}$-Lösung, mit 5-proz. wäßriger Natronlauge und zuletzt mehrmals mit destilliertem Wasser ausgewaschen. Nach Trocknung mit Sikkon und mit Molekularsieb $4 \AA$ wurde der Äther über eine 10-bödige automatische

1 F. Becker, H. D. Pflug u. M. Kiefer, Z. Naturforsch. 23 a, 1805 [1968].

${ }^{2}$ F. Becker, E. W. Fries, M. Kiefer u. H. D. Pflug, Z. Naturforsch. 25 a, 677 [1970].

${ }^{3}$ F. BeCKer u. M. Kiefer, Z. Naturforsch. 24 a, 7 [1969].

4 M. KIEFER, Dissertation, Saarbrücken 1969. 
Vigreux-Kolonne feinfraktioniert. Bei Propylenoxid entfiel wegen der Hydrolyseempfindlichkeit die Behandlung mit $\mathrm{KMnO}_{4}$ und mit $\mathrm{NaOH}$; bei den mit Wasser mischbaren Äthern wurde zwischen die einzelnen Waschoperationen jeweils eine einfache Destillation eingeschaltet, um die wäßrige Lösung abzutrennen.

Diäthyläther :

$$
K_{p_{735}} 33,6^{\circ} ; \quad n_{\mathrm{D}}^{19,2} 1,3530 ; \quad D_{4}^{25} 0,7076 .
$$

Di-n-propyläther :

$$
n_{\mathrm{D}}^{17,9} 1,3812 ; \quad D_{4}^{25} 0,7427 .
$$

Di-n-butyläther :

$$
K_{p 738} 141,3^{\circ} ; \quad n_{\mathrm{D}}^{25} \quad 1,3976 ; D_{4}^{25} 0,7643 .
$$

Di-isopropyläther :

$$
K_{p 740} \quad 67,5^{\circ} ; \quad n_{\mathrm{D}}^{25} \quad 1,3662 ; \quad D_{4}^{25} 0,7186 .
$$

Propylenoxid:

$$
K_{p 725} \quad 32,9^{\circ} ; \quad n_{\mathrm{D}}^{20} \quad 1,3664 ; \quad D_{4}^{25} \quad 0,8222 .
$$

Tetrahydrofuran:

$$
K_{p 740} \quad 65,3^{\circ} ; \quad n_{\mathrm{D}}^{25} \quad 1,4052 ; \quad D_{4}^{25} 0,8825 .
$$

Tetrahydropyran:

$$
K_{p 740} \quad 87,1^{\circ} ; \quad n_{\mathrm{D}}^{25} \quad 1,4190 ; \quad D_{4}^{25} 0,8797 .
$$

Zur Prüfung der Frage, ob wegen der auftretenden Volumenkontraktionen eine Korrektur der $\mathrm{Zu}$ laufgeschwindigkeiten erforderlich ist ${ }^{3}$, haben wir die Exzeßvolumina $V^{\mathrm{E}}$ beim Molenbruch 0,5 pyknometrisch ermittelt. Sie betragen bei $25^{\circ}$ für die Äther - Chloroform-Systeme in der oben angegebenen Reihenfolge: $-1,4 \pm 0,2 ;-0,7 \pm 0,2 ;-0,2$ $\pm 0,2 ;-1,3 \pm 0,2 ; \pm 0,0 \pm 0,2 ;-0,3 \pm 0,2$ und $-0,3 \pm 0,2 \mathrm{ml} / \mathrm{Mol}$. Demnach konnten bei den Systemen mit Di-n-butyläther sowie mit den drei cyclischen Äthern Korrekturen unterbleiben, während bei den übrigen Systemen der Einfluß der Volumenkontraktion in den Auswerteformeln in erster Näherung berücksichtigt wurde.

\section{b) Integrale Mischungswärmen}

Tabelle 1 enthält eine Zusammenstellung der Funktionswerte $H^{\mathrm{M}}=f\left(N_{\mathrm{CHCl}_{3}}\right)$ bei $25^{\circ}$ für die 7 untersuchten Äther-Chloroform-Systeme. Angegeben sind die Mittelwerte von jeweils 2-3 Messungen, die untereinander innerhalb von $1 \%$ übereinstimm. ten. Eine weitere Kontrolle für die Richtigkeit von Messung und Auswertung war dadurch gegeben, daß die beiden, bei Vorlage von reinem $̈$ ther bzw. reinem Chloroform erhaltenen Kurvenäste mit einer Abweichung von höchstens $1 \%$ ineinander übergingen.
In Abb. 1 sind die Funktionen $H^{\mathrm{M}}=f\left(N_{\mathrm{CHCls}}\right)$ für die 4 aliphatischen, in Abb. 2 für die drei cyclischen Äther graphisch wiedergegeben. Während die Kurven in Abb. 1 weitgehend symmetrisch sind, weisen sie in Abb. 2 eine deutliche Unsymmetrie mit Verschiebung des Extremwertes aus der Mitte zu hö-

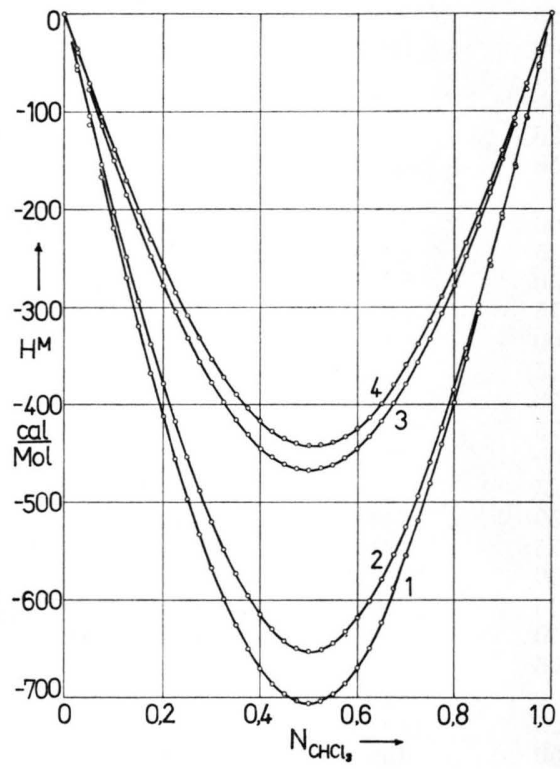

Abb. 1. Funktionen $H^{\mathrm{M}}=f\left(\mathrm{~N}_{\mathrm{CHCl}_{3}}\right)$ bei $25^{\circ}$. 1) $\mathrm{iPr}_{2} \mathrm{O}-\mathrm{CHCl}_{3}$;2) $\left.\mathrm{Et}_{2} \mathrm{O}-\mathrm{CHCl}_{3} ; 3\right) \mathrm{nPr}_{2} \mathrm{O}-\mathrm{CHCl}_{3}$; 4) $\mathrm{nBu}_{2} \mathrm{O}-\mathrm{CHCl}_{3}$.

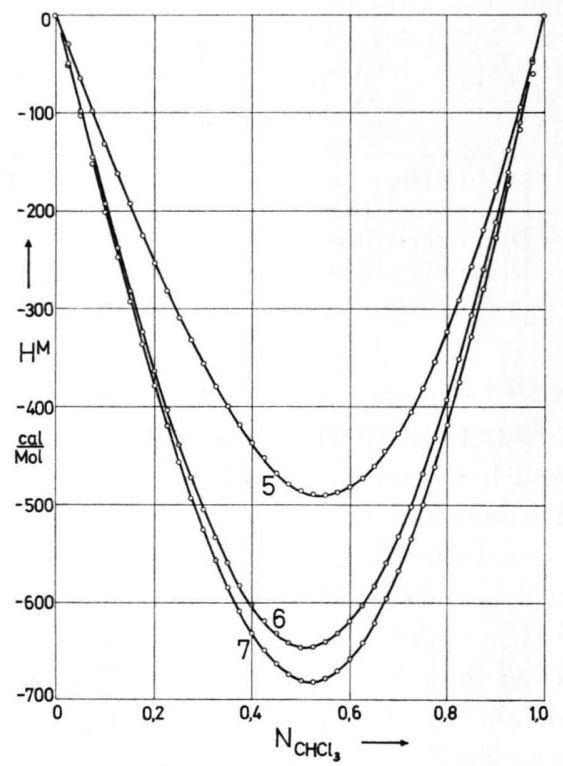

Abb. 2. Funktionen $H \mathrm{M}=f\left(\mathrm{~N}_{\mathrm{CHCl}_{3}}\right)$ bei $25^{\circ}$. 5) Propylenoxid- $\mathrm{CHCl}_{3}$; 6) Tetrahydropyran $-\mathrm{CHCl}_{3}$; 7) Tetrahydrofuran $-\mathrm{CHCl}_{3}$. 
Tab. 1. Integrale Mischungswärmen der Systeme Äther-Chloroform.

\begin{tabular}{|c|c|c|c|c|c|c|c|}
\hline & & & $-H^{\mathrm{M}}[\mathrm{ce}$ & bei $\left.25^{\circ}\right]$ & & & \\
\hline $\mathrm{NCHCl}_{3}$ & $\mathrm{EtO}_{2}$ & $\mathrm{nPr}_{2} \mathrm{O}$ & $\mathrm{nBu}_{2} \mathrm{O}$ & $\mathrm{iPr}_{2} \mathrm{O}$ & $\mathrm{PrOx}$ & $\mathrm{THF}$ & THPy \\
\hline 0,025 & 52,6 & 39,2 & 35,9 & 56,7 & 29,3 & 51,9 & 49,1 \\
\hline 0,050 & 104,0 & 77,2 & 71,0 & 112,4 & 64,2 & 102,5 & 97,7 \\
\hline 0,075 & 154,1 & 114,1 & 105,2 & 166,5 & 97,8 & 152,0 & 145,5 \\
\hline 0,100 & 202,2 & 149,4 & 138,3 & 219,1 & 131,4 & 200,2 & 192,1 \\
\hline 0,125 & 248,8 & 184,0 & 170,1 & 270,0 & 161,8 & 247,0 & 237,4 \\
\hline 0,150 & 293,8 & 216,7 & 200,8 & 319,3 & 192,2 & 292,3 & 281,2 \\
\hline 0,175 & 337,1 & 247,8 & 230,1 & 367,8 & 224,4 & 336,0 & 323,4 \\
\hline 0,200 & 377,7 & 277,6 & 257,9 & 411,9 & 253,4 & 378,0 & 363,8 \\
\hline 0,225 & 416,6 & 305,3 & 284,2 & 454,8 & 281,0 & 418,1 & 402,3 \\
\hline 0,250 & 453,2 & 331,5 & 308,8 & 496,2 & 308,5 & 456,2 & 438,5 \\
\hline 0,275 & 487,6 & 355,5 & 331,7 & 532,5 & 330,6 & 492,1 & 472,7 \\
\hline 0,300 & 519,3 & 377,4 & 352,7 & 566,8 & 355,8 & 525,4 & 504,2 \\
\hline 0,325 & 548,0 & 397,3 & 371,8 & 598,1 & 378,1 & 556,3 & 533,1 \\
\hline 0,350 & 573,1 & 414,9 & 388,7 & 625,7 & 399,4 & 584,2 & 559,0 \\
\hline 0,375 & 595,6 & 430,1 & 403,5 & 649,6 & 418,0 & 609,0 & 582,2 \\
\hline 0,400 & 614,4 & 442,8 & 416,0 & 669,7 & 436,4 & 630,6 & 602,0 \\
\hline 0,425 & 629,9 & 452,8 & 426,1 & 685,5 & 451,5 & 648,7 & 618,5 \\
\hline 0,450 & 641,8 & 460,2 & 433,8 & 696,8 & 467,1 & 663,0 & 631,2 \\
\hline 0,475 & 649,1 & 464,7 & 438,9 & 703,6 & 478,1 & 673,1 & 640,1 \\
\hline 0,500 & 652,6 & 466,5 & 441,4 & 705,9 & 485,4 & 678,4 & 645,1 \\
\hline 0,525 & 651,4 & 465,6 & 441,2 & 703,6 & 489,3 & 681,0 & 646,3 \\
\hline 0,550 & 644,0 & 461,7 & 438,3 & 696,6 & 490,0 & 677,6 & 643,6 \\
\hline 0,575 & 631,8 & 455,0 & 432,7 & 685,0 & 487,4 & 669,6 & 630,6 \\
\hline 0,600 & 618,4 & 445,5 & 424,4 & 668,9 & 481,7 & 657,3 & 618,6 \\
\hline 0,625 & 600,7 & 432,8 & 413,4 & 648,9 & 472,7 & 640,9 & 602,6 \\
\hline 0,650 & 578,9 & 416,2 & 399,8 & 623,0 & 460,5 & 620,2 & 582,7 \\
\hline 0,675 & 554,0 & 398,4 & 380,0 & 589,4 & 445,1 & 595,7 & 559,1 \\
\hline 0,700 & 525,5 & 378,7 & 358,0 & 554,7 & 426,8 & 567,3 & 532,0 \\
\hline 0,725 & 494,1 & 356,6 & 337,5 & 519,2 & 405,3 & 535,4 & 501,5 \\
\hline 0,750 & 459,5 & 332,5 & 314,7 & 481,1 & 380,9 & 500,0 & 468,0 \\
\hline 0,775 & 423,6 & 306,2 & 289,7 & 440,5 & 353,8 & 461,3 & 431,5 \\
\hline 0,800 & 384,3 & 278,1 & 263,0 & 397,7 & 323,9 & 419,7 & 392,3 \\
\hline 0,825 & 342,3 & 248,2 & 234,5 & 352,9 & 291,4 & 375,2 & 350,5 \\
\hline 0,850 & 298,2 & 216,6 & 204,4 & 306,1 & 256,4 & 328,2 & 306,2 \\
\hline 0,875 & 252,6 & 183,5 & 172,9 & 258,0 & 219,1 & 278,7 & 259,8 \\
\hline 0,900 & 204,7 & 149,1 & 140,1 & 208,4 & 179,5 & 226,9 & 211,2 \\
\hline 0,925 & 156,0 & 113,6 & 106,3 & 157,8 & 137,6 & 173,1 & 160,8 \\
\hline 0,950 & 105,0 & 76,8 & 71,6 & 106,1 & 93,7 & 117,2 & 108,7 \\
\hline 0,975 & 52,5 & 38,9 & 36,2 & 53,6 & 47,8 & 59,4 & 55,0 \\
\hline $\mathrm{Et}_{2} \mathrm{O}=\mathrm{D}$ & her & & $=$ Propyle & & & & \\
\hline$n \mathrm{Pr}_{2} \mathrm{O}=\mathrm{D}$ & pyläther & & $=$ Tetrahy & & & & \\
\hline $\begin{aligned} \mathrm{nBu}_{2} \mathrm{O} & =\mathrm{D} \\
\mathrm{iPr}_{2} \mathrm{O} & =\mathrm{D}\end{aligned}$ & $\begin{array}{l}\text { yläther } \\
\text { opyläther }\end{array}$ & & $=$ Tetrahy & & & & \\
\hline
\end{tabular}

heren $\mathrm{CHCl}_{3}$-Konzentrationen und einer größeren Grenzsteigung auf der chloroformreichen Seite auf. Viel deutlicher kommt der Unterschied zwischen den symmetrischen und unsymmetrischen Mischungskurven in der Darstellung $H^{\mathrm{M}}=f\left[N(1-N) /\left(-H^{\mathrm{M}}\right)\right]$ zum Ausdruck. Die entsprechenden Kurven für die aliphatischen Äther sind in Abb. 3, für die cyclischen Äther in Abb. 4 wiedergegeben. Tabelle 2 enthält die Zahlenwerte für die Minima und die Grenzsteigungen der Funktionen $H^{\mathrm{M}}=f\left(N_{\mathrm{CHCl}_{3}}\right)$ sowie für die Steigungen der Grenztangenten der Funktionen $H^{\mathrm{M}}=f\left[N(1-N) /\left(-H^{\mathrm{M}}\right)\right]$, die ebenfalls den Un- terschied zwischen den symmetrischen und den unsymmetrischen Kurven erkennen lassen.

Bestimmungen der integralen Mischungswärmen von aliphatischen Äthern mit Chloroform bei $25^{\circ}$ wurden in jüngster Zeit von BeAth und Williamson ${ }^{5}$ durchgeführt. Diese Autoren haben den Verlauf der Mischungskurven (jeweils 10-12 Meßwerte) durch den 5-parametrigen erweiterten Porterschen Ansatz

$$
H^{\mathrm{M}}=N(1-N) \sum_{i=0}^{4} A_{i}(1-2 N)^{i}
$$

5 L. A. Beath u. A. G. Williamson, J. Chem. Thermodynamics 1, 51 [1969]. 


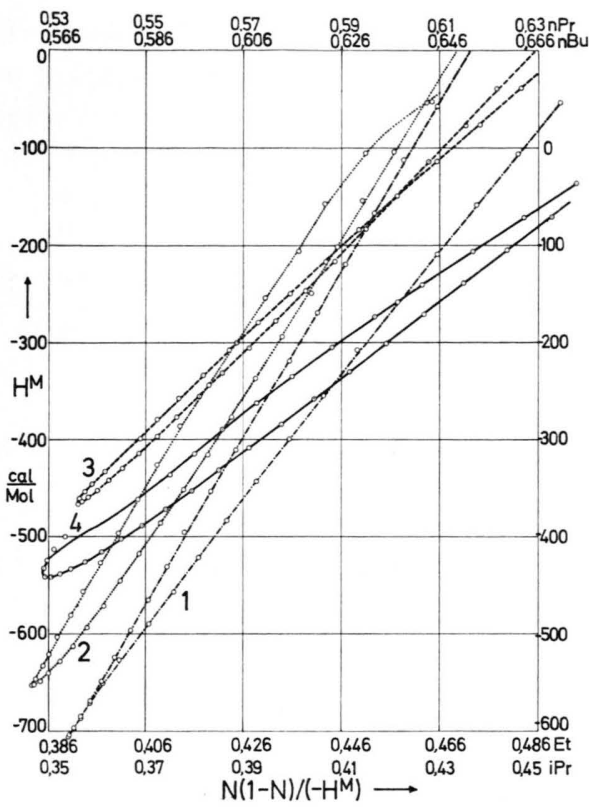

Abb. 3. Funktionen $H^{\mathrm{M}}=f\left[N(1-N) /\left(-H^{\mathrm{M}}\right)\right]$ bei $25^{\circ}$ 1) $\left.(-\cdot-\cdot-) \mathrm{iPr}_{2} \mathrm{O}-\mathrm{CHCl}_{3} ; 2\right)(\cdots \cdots) \mathrm{Et}_{2} \mathrm{O}-\mathrm{CHCl}_{3}$; 3) $\left.(----) \mathrm{nPr}_{2} \mathrm{O}-\mathrm{CHCl}_{3} ; 4\right)(-) \mathrm{nBu}_{2} \mathrm{O}-\mathrm{CHCl}_{3}$. Zur Kurve 4) gehört der Ordinatenmaßstab auf der rechten Seite.

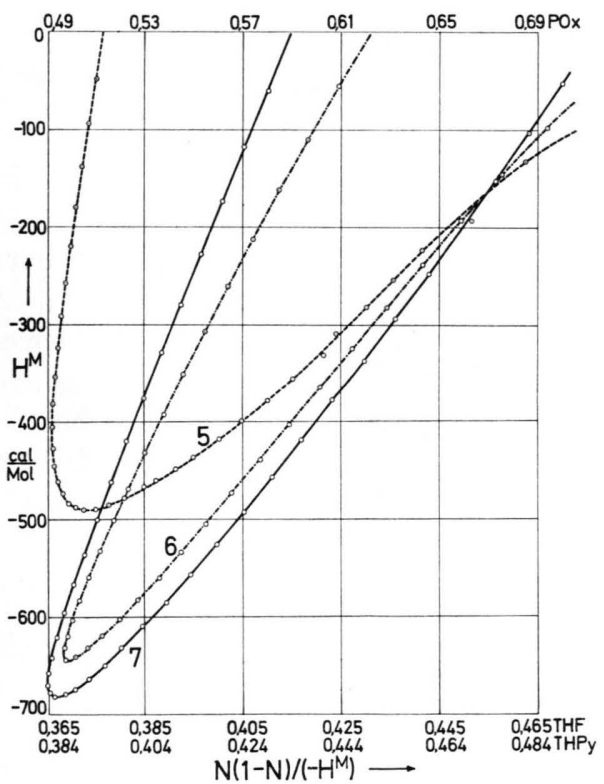

Abb. 4. Funktionen $H^{\mathrm{M}}=f\left[N(1-N) /\left(-H^{\mathrm{M}}\right)\right]$ bei $25^{\circ}$. 5) (- - - ) Propylenoxid- $\left.\mathrm{CHCl}_{3} ; 6\right)(-.-.-)$ Tetrahydropyran $\left.-\mathrm{CHCl}_{3} ; 7\right)(\longmapsto)$ Tetrahydrofuran $-\mathrm{CHCl}_{3}$.

wiedergegeben. Vergleicht man die mit den Parameterwerten $A_{i}$ der Autoren berechneten Funktionen $H^{\mathrm{M}}=$ $f\left(N_{\mathrm{CHCl}_{3}}\right)$ mit unseren Ergebnissen, so verlaufen die nach Gl. (1) berechneten Kurven zu beiden Seiten des
Extremwertes etwas flacher, dafür an den Rändern steiler als die Kurven in Abb. 1 . Im übrigen ist die Übereinstimmung bei den Systemen $\mathrm{nPrO}_{2}-\mathrm{CHCl}_{3}$ und $\mathrm{nBu}_{2} \mathrm{O}-\mathrm{CHCl}_{3}$ am besten. Beath und Williamson finden für das System $\mathrm{Et}_{2} \mathrm{O}-\mathrm{CHCl}_{3}$ einen Extremwert, der um $20 \mathrm{cal} / \mathrm{Mol}$ höher liegt als unser Resultat; auch der ganze chloroformreiche Ast liegt deutlich oberhalb des von uns gefundenen Kurvenverlaufes, während alle von $\mathrm{HIROBE}^{6}$ angegebenen Meßwerte innerhalb von $1 \%$ mit unseren übereinstimmen. Beim System $\mathrm{iPr}_{2} \mathrm{O}-$ $\mathrm{CHCl}_{3}$ besteht Übereinstimmung in der Nähe des Extremwertes, während die Kurvenform in der oben angegebenen Weise von der in Abb. 1 abweicht. Bei den Systemen $\mathrm{Et}_{2} \mathrm{O}-\mathrm{CHCl}_{3}$ und $\mathrm{iPr}_{2} \mathrm{O}-\mathrm{CHCl}_{3}$ weisen die nach den Messungen von Beath und Williamson mittels Gl. (1) berechneten Grenzsteigungen $m_{0}$ und $m_{1}$ Unterschiede von $20-25 \%$ auf, wobei die Unsymmetrie im letzteren Fall gerade umgekehrt liegt, wie von uns gefunden wurde. Offenbar ist eine durch die Meßwerte gelegte Ausgleichskurve vom Typ der Gl. (1) weniger gut geeignet, um das Verhalten der Mischungswärmen an den Grenzen des Konzentrationsbereiches richtig wiederzugeben, als die von uns benützten Gleichungen des Ein- bzw. Zweistufen-Gleichgewichtsmodells.

\section{Interpretation der Meßergebnisse durch Gleichgewichtsmodelle}

Wie in ${ }^{1}$ gezeigt wurde, sollte bei Vorliegen eines Einstufengleichgewichtes vom Typ $\mathrm{A}+\mathrm{B}=\mathrm{AB}$ dit Funktion $H^{\mathrm{M}}=f\left[\mathrm{~N}(1-N) /\left(-H^{\mathrm{M}}\right)\right]$ die Form einer Geraden mit dem Ordinatenabschnitt $H_{\mathrm{AB}}$ und der Steigung $H_{\mathrm{AB}}^{2} K_{1} /\left(1+K_{1}\right)$ besitzen. Aus Abb. 3 geht hervor, daß die Mischungen der drei unverzweigten aliphatischen Äther mit Chloroform diese Bedingung weitgehend erfüllen. Da diese Art der Darstellung Unsymmetrien gegenüber äußerst empfindlich ist, kann insbesondere die Tatsache, daß die beiden Kurvenäste an den Grenzen des Konzentrationsbereiches einander wieder näherkommen, als wesentliches Kriterium für die Anwendbarkeit des Einstufen-Gleichgewichtsmodells angesehen werden. In diesen Fällen rührt also die gemessene Mischungswärme fast ausschließlich von der Bildung einer $\mathrm{H}$ Brückenverbindung im Molverhältnis 1: 1 zwischen der $\mathrm{C}-\mathrm{H}$-Bindung des Chloroforms und einem der einsamen Elektronenpaare des Äthersauerstoffs her.

Beim System Diisopropyläther - Chloroform streben die beiden Kurvenäste (Abb. 3) an den Grenzen des Meßbereiches auseinander, wobei - im Gegensatz zu allen übrigen Systemen - der chloroformarme Ast die größere Steilheit besitzt. Hier lie-

${ }^{6}$ H. Hirobe, J. Fac. Sci. Imperial Univ. Tokyo, Sect. I, Vol. 1, 156 [1925-29]. 
gen offensichtlich geringfügige Abweichungen vom Einstufen-Gleichgewichtsmodell vor, für die aber zum gegenwärtigen Zeitpunkt eine Deutung nicht gegeben werden kann.

Bei den cyclischen Äthern zeigen die Funktionen $H^{\mathrm{M}}=f\left[N(1-N) /\left(-H^{\mathrm{M}}\right)\right](\mathrm{Abb} .3)$ einen hyperbelähnlichen Verlauf, wobei die Öffnung der Kurven mit wachsender Ringgröße des Äthers abnimmt. Eine Anwendung des Einstufen-Gleichgewichtsmodells ist damit ausgeschlossen. Prinzipiell ist aber die Bildung einer $1: 2$-Verbindung des Äthers mit 2 Molekülen Chloroform möglich, da der Äthersauerstoff zwei einsame Elektronenpaare besitzt. Im System Tetrahydrofuran-Chloroform konnte die Bildung einer solchen $1: 2$-Verbindung auch durch dielektrische Messungen nachgewiesen werden ${ }^{7}$.

Wie in ${ }^{2}$ gezeigt worden ist, läßt sich der Verlauf der Grenztangenten der Funktion

$$
H^{\mathrm{M}}=f\left[N(1-N) /\left(-H^{\mathrm{M}}\right)\right]
$$

näherungsweise durch die Gleichungen

$$
\begin{gathered}
H^{\mathrm{M}}=H_{\mathrm{AB}}+m_{0}{ }^{*} N(1-N) /\left(-H^{\mathrm{M}}\right) \\
\left(\mathrm{CHCl}_{3} \text {-armer Ast }\right), \\
H^{\mathrm{M}}=H_{\mathrm{AB}_{2}+m_{1}{ }^{*} N(1-N) /\left(-H^{\mathrm{M}}\right)} \\
\left(\mathrm{CHCl}_{3} \text {-reicher Ast }\right)
\end{gathered}
$$

wiedergeben, wobei die Steigungen

$$
m_{0}{ }^{*}=\left(-m_{1}\right) H_{\mathrm{AB}} \text { und } m_{1}{ }^{*}=m_{0} H_{\mathrm{AB}_{2}}
$$

lauten $\left(H_{\mathrm{AB}_{2}}\right.$ ist die Brutto-Reaktionsenthalpie für die Bildung von $\mathrm{AB}_{2}$ ) und

$$
\begin{aligned}
& m_{0}=\left(H_{\mathrm{AB}} K_{1}+H_{\mathrm{AB}_{2}} K_{1} K_{2}\right) /\left(1+K_{1}+K_{1} K_{2}\right) ; \\
& m_{1}=-H_{\mathrm{AB}} K_{1} /\left(1+K_{1}\right)
\end{aligned}
$$

die Grenzsteigungen der Funktion $H^{\mathrm{M}}=f\left(\mathrm{~N}_{\mathrm{CHCl}_{3}}\right)$ sind. Praktisch erlaubt es der experimentell gefundene Verlauf der in Abb. 4 wiedergegebenen Funk- tionen, die Steigungen der Grenztangenten mit einiger Sicherheit zu bestimmen, da die Kurven an den Grenzen des Konzentrationsbereiches fast geradlinig werden. Aus den Steigungen und den Ordinatenabschnitten dieser Grenztangenten erhält man unter Benützung von $m_{0}$ und $m_{1}$ nach Gl. (5) alle 4 Parameter der Zweistufen-Gleichgewichte. Tabelle 3 enthält eine Zusammenstellung der auf diese Weise graphisch ermittelten thermodynamischen Gleichgewichtsdaten der 7 untersuchten Äther-ChloroformSysteme. Bei den als Einstufen-Gleichgewichte behandelten Systemen Di-n-alkyläther-Chloroform wurden Ordinatenabschnitt und Steigung der durch die schleifenförmigen Kurven (Abb. 3) gelegten Mittelgeraden verwendet. Im Falle des Systems Diisopropyläther-Choroform wurden die Gleichgewichtsdaten aus dem Verlauf des chloroformarmen Astes berechnet in der Annahme, daß dieser Ast von den vorhandenen Abweichungen vom EinstufenGleichgewichtsmodell weniger betroffen ist.

Eine genauere Betrachtung des Verlaufes der Kurven in Abb. 4 zeigt, daß der chloroformarme Ast des Systems Tetrahydrofuran - Chloroform von $N_{\mathrm{CHCl}_{3}}<0,1$ an eine leichte Rechtskrümmung aufweist. Da dieses Verhalten offensichtlich von einer Abweichung vom Zweistufen-Gleichgewichtsmodell herrührt, wurden die ersten drei Meßwerte bei der Bestimmung der Steigung der „Grenztangente“ nicht berücksichtigt. Noch deutlicher macht sich die Abkrümmung am Ende des chloroformarmen Astes im System Propylenoxid - Chloroform bemerkbar. Hierfür ist eine beim Propylenoxid im Gegensatz zu den

\begin{tabular}{|c|c|c|c|c|c|c|}
\hline Äther & $N_{\mathrm{CHC} 13}^{\max }$ & $-H_{\max }^{\mathrm{M}}$ & $\begin{array}{l}-m_{0} \\
\quad[\mathrm{kcal} / \mathrm{Mol}]\end{array}$ & $m_{1}$ & $m_{0}$ & $m_{1}$ \\
\hline $\mathrm{Et}_{2} \mathrm{O}$ & 0,505 & 0,653 & 2,11 & 2,14 & & \\
\hline $\mathrm{nPr}_{2} \mathrm{O}$ & 0,502 & 0,467 & 1,53 & 1,53 & & \\
\hline $\mathrm{nBu}_{2} \mathrm{O}$ & 0,505 & 0,442 & 1,43 & 1,43 & & \\
\hline $\mathrm{iPr}_{2} \mathrm{O}$ & 0,500 & 0,706 & 2,24 & 2,17 & 6,28 & 8,65 \\
\hline $\mathrm{PrOx}$ & 0,546 & 0,490 & 1,32 & 1,90 & 2,50 & 14,8 \\
\hline THF & 0,522 & 0,681 & 2,04 & 2,32 & 6,75 & 12,4 \\
\hline THPy & 0,508 & 0,647 & 1,94 & 2,16 & 6,06 & 9,49 \\
\hline
\end{tabular}
übrigen Äthern deutlich vorhandene Eigenassoziation verantwortlich zu machen. Diese Eigenassoziation kommt in dem stark unsymmetrischen Verlauf der Funktion $H^{\mathrm{M}}=f\left(N_{\mathrm{CCl}_{4}}\right)$ bei $25^{\circ}$ des Systems Propylenoxid-Tetrachlorkohlenstoff ${ }^{8}$ zum Aus-

Tab. 2. Systeme Äther-Chloroform bei $25^{\circ}$. Extremwerte der Funktionen $H^{\mathrm{M}}=f\left(N_{\mathrm{CHCl}_{3}}\right)$; Grenzwerte $m_{0}$ und $m_{1}$ der differentiellen Mischungswärmen; Steigungen $m_{0}{ }^{*}$ und $m_{1}{ }^{*}$ der Grenztangenten der Funktionen $H^{\mathrm{M}}=f\left[N(1-N) /\left(-H^{\mathrm{M}}\right)\right]$.

7 A. Weisbecker, J. Chim. Physique 62, 1170 [1965]. - J. Barriol u. A. Weisbecker, J. Chim. Physique 62, 623 [1965]. 8 H. Koukol, Dissertation, Saarbrücken 1970. 
druck, die bei hohen $\mathrm{CCl}_{4}$-Konzentrationen sogar das Vorzeichen wechselt. Bei der Bestimmung der Steigung $m_{0}{ }^{*}$ des Systems Propylenoxid-Chloroform wurden deshalb die Meßwerte im Bereich $N_{\mathrm{CHCl}_{3}}<0,125$, in denen der Einfluß der Eigenassoziation des Propylenoxids am stärksten bemerkbar wird, nicht berücksichtigt.

Aus der symmetrischen und fast exakt parabelförmigen Form der Funktionen $H^{\mathrm{M}}=f\left(\mathrm{~N}_{\left(\mathrm{CCl}_{4}\right)^{8}}\right.$ von Systemen aus aliphatischen Äthern und Tetrachlorkohlenstoff kann gefolgert werden, daß beide Mischungskomponenten keine merkliche Eigenassoziation aufweisen.
Wie in ${ }^{1}$ gezeigt wurde, kann bei assoziierten Flüssigkeiten nur dann eine symmetrische Mischungskurve resultieren, wenn Assoziationstyp und thermodynamische Gleichgewichtsdaten bei beiden Komponenten weitgehend identisch sind. Der ebenfalls symmetrische und parabelförmige Verlauf der Mischungskurve des Systems $\mathrm{CHCl}_{3}-\mathrm{CCl}_{4}{ }^{9}$ beweist ferner, daß auch diese beiden Flüssigkeiten bei $25^{\circ}$ nicht zu spezifischer Eigenassoziation neigen. Außerdem geht aus dem Vergleich der Verdampfungsenthalpien der Äther und strukturanaloger Kohlenwasserstoffe (Ersatz von - $\mathrm{O}$ - durch $-\mathrm{CH}_{2}-$ ) hervor, daß aliphatische Äther nicht nennenswert assoziiert sein können:

Verdampfungsenthalpien in kcal/Mol bei $298{ }^{\circ} \mathrm{K}^{10}$ :

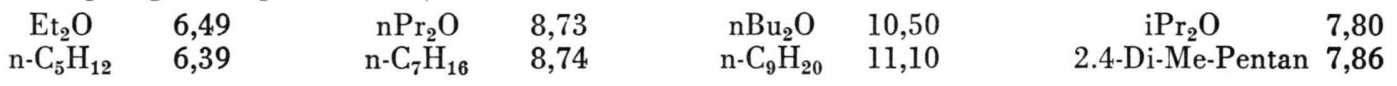

Dagegen haben die cyclischen Äther gegenüber den strukturanalogen Kohlenwasserstoffen merklich erhöhte Verdampfungsenthalpien, wobei der Unterschied mit wachsender Ringgröße abnimmt:
Propylenoxid
6,67
(Cyclobutan)
$(5,89)$
Tetrahydrofuran
7,65
6,85
Tetrahydropyran
8,35
Cyclopentan
Cyclohexan
7,90

Tab. 3. Thermodynamische Daten der Systeme Äther (A) - Chloroform (B) bei $25^{\circ}$, aus den graphisch bestimmten Werten $m_{0}, m_{1}, m_{0}{ }^{*}, m_{1}{ }^{*}$ nach dem Einstufen- bzw. Zweistufen-Gleichgewichtsmodell berechnet. Stufe 1: $\mathrm{A}+\mathrm{B}=\mathrm{AB} ;$ Stufe $2: \mathrm{AB}+\mathrm{B}=\mathrm{AB}_{2}$.

\begin{tabular}{|c|c|c|c|c|c|c|c|c|}
\hline Äther & $K_{1}$ & $K_{2}$ & $-H_{A B}^{(1)}$ & $\begin{array}{l}-H_{\mathrm{AB}}^{(2)} \\
{[\mathrm{kcal} / \mathrm{Mol}]}\end{array}$ & $-G_{\mathrm{AB}}^{(1)}$ & $-G_{\mathrm{AB}}^{(2)}$ & $\begin{array}{l}-S_{\mathrm{AB}}^{(1)} \\
\quad[\mathrm{cal} / \mathrm{Mol} .\end{array}$ & $\begin{array}{l}-S_{\mathrm{AB}}^{(2)} \\
\text { Grad] }\end{array}$ \\
\hline $\mathrm{Et}_{2} \mathrm{O}$ & 1,42 & & 3,65 & & 0,208 & & 11,5 & \\
\hline $\mathrm{nPr}_{2} \mathrm{O}$ & 1,06 & & 3,07 & & 0,035 & & 10,2 & \\
\hline $\mathrm{nBu}_{2} \mathrm{O}$ & 1,34 & & 2,55 & & 0,173 & & 8,0 & \\
\hline $\mathrm{iPr}_{2} \mathrm{O}$ & 1,55 & & 3,77 & & 0,260 & & 11,8 & \\
\hline PrOx & $(2,84)$ & $(0,13)$ & $(1,83)$ & 5,79 & $(0,618)$ & $(1,21)$ & $(4,1)$ & $(23)$ \\
\hline THF & 1,83 & 0,16 & 3,23 & 1.93 & 0,358 & $(1,09$ & 9,6 & 10,1 \\
\hline THPy & 1,94 & 0,12 & 3,03 & 1,23 & 0,393 & 1,28 & 8,8 & 8,4 \\
\hline
\end{tabular}

Wie aus Tab. 3 hervorgeht, nehmen die Beträge der Reaktionsenthalpien $H_{\mathrm{AB}}$ innerhalb der Reihe der aliphatischen Äther mit wachsender Kettenlänge ab. Bei der Deutung dieses Effektes muß beachtet werden, daß $H_{\mathrm{AB}}$ die Reaktionsenthalpie für die Bildung der Verbindung $\mathrm{AB}$ aus den reinen Flüssigkeiten A und B darstellt und somit in gewissem Umfang von den in A und B vorhandenen intermolekularen Wechselwirkungsenergien abhängig ist. Als $\mathrm{Ma}$ hierfür kann die Verdampfungsenthalpie $\Delta H_{\mathrm{v}}$ der betreffenden Flüssigkeit, ebenfalls bei $298{ }^{\circ} \mathrm{K}$ gemessen, angesehen werden. Tatsächlich findet man zwischen den Verdampfungsenthalpien homologer

9 M. L. McGlashan, J. E. Prue u. I. E. J. Sainsbury, Trans. Faraday Soc. 50, 1284 [1954]. - D. A. ADCock u. M. L. McGlashan, Proc. Roy. Soc. London A 226, 266 [1954].
Äther und den Grenzwerten $m_{1}$ ihrer differentiellen Mischungsenthalpien mit Chloroform einen genähert linearen Zusammenhang. Aus den wenigen verfügbaren Meßdaten kann man entnehmen, daß eine Zunahme der Verdampfungsenthalpie um $\delta \Delta H_{\mathrm{v}}$ bei Übergang von Diäthyläther zu einem höheren homologen, unverzweigten Äther mit einem Gewichtsfaktor von rund 0,2 in die Grenzsteigung $m_{1}$ der Mischungskurve mit Chloroform eingeht. Nimmt man an, daß die Änderungen $\delta \Delta H_{\mathrm{v}}$ nur die Reaktionsenthalpien $H_{\mathrm{AB}}$ und nicht die Reaktionsentropien $S_{\mathrm{AB}}$ betreffen, so läßt sich der Einfluß der Verdampfungsenthalpien auf $H_{\mathrm{AB}}$ durch Subtraktion

10 J. D. Cox u. G. PIlcher, Thermochemistry of Organic and Organometallic Compounds, Academic Press, New York 1970. 
von $0,2 \cdot \delta \Delta H_{\mathrm{v}}$ korrigieren, wobei die Daten für das Anfangsglied der homologen Reihe als Bezugsgröße dienen. Durch diese Korrektur werden die $H_{\mathrm{AB}}$ Werte der Systeme $\mathrm{nPr}_{2} \mathrm{O}-\mathrm{CHCl}_{3}, \mathrm{nBu}_{2} \mathrm{O}-\mathrm{CHCl}_{3}$ $\left(\mathrm{Et}_{2} \mathrm{O}\right.$ als Bezugswert) und THPy $-\mathrm{CHCl}_{3}$ (THF als Bezugswert) auf $-3,32$; $-3,35$ bzw. $-3,17$ kcal/Mol gebracht, so daß die in Tab. 3 auftretenden Unterschiede weitgehend verschwinden. Daß für das System $\mathrm{iPr}_{2} \mathrm{O}-\mathrm{CHCl}_{3}$ ein größerer Betrag von $H_{\mathrm{AB}}$ gefunden wird, kann als Folge der erhöhten induktiven Wechselwirkung zwischen der polaren $\mathrm{C}-\mathrm{H} \ldots \mathrm{O}$-Bindung und der stärker polarisierbaren Isopropylgruppe gedeutet werden.

Für das System Propylenoxid - Chloroform sind in Tab. 3 die durch formale Anwendung des Zweistufen-Gleichgewichtsmodells errechneten thermodynamischen Daten in Klammern gesetzt, weil in diesem Fall das Modell angesichts der stark von den übrigen abweichenden Resultate möglicherweise nicht zutrifft.

Die Feststellung, daß nur bei den cyclischen Äthern ein zweite Gleichgewichtsstufe in Erscheinung tritt, hat sehr wahrscheinlich sterische Ursachen. Die Annäherung eines zweiten Chloroformmoleküls an den Äthersauerstoff auf den zur Ausbildung einer H-Brücke erforderlichen Abstand wird von den Alkylresten offenbar nur dann nicht behindert, wenn diese in einer weitgehend ebenen Ringstruktur fixiert sind.

Die Deutsche Forschungsgemeinschaft, Bad Godesberg, hat die vorliegende Arbeit durch Sachbeihilfen unterstützt, wofür wir auch an dieser Stelle unseren Dank aussprechen.

\title{
Thermodynamik heterogener Gasgleichgewichte
}

\section{Freie Bildungsenthalpie $\Delta G_{\mathrm{B}}^{0}$ der Wolframoxihalogenverbindungen}

\author{
G. M. Neumann und W. KnatZ \\ OSRAM-Studiengesellschaft München
}

(Z. Naturforsch. 26 a, 1046-1053 [1971] ; eingegangen am 24. März 1971)

\begin{abstract}
Zur thermodynamischen Berechnung der chemischen Transportreaktionen in den Wolfram-Halogen-Sauerstoff-Systemen werden auf Grund empirischer Betrachtungen der Bindungsverhältnisse in den Molekülen die freien Bildungsenthalpien $\Delta G_{\mathrm{B}}^{0}$ der Verbindungen $\mathrm{WO}_{2} \mathrm{Br}_{2}, \mathrm{WOBr}_{4}, \mathrm{WO}_{2} \mathrm{~J}_{2}$ und der noch hypothetischen Verbindungen $\mathrm{WO}_{2} \mathrm{~F}_{2}$ und $\mathrm{WOJ}_{4}$ für Temperaturen zwischen 500 und $3600 \mathrm{~K}$ ermittelt. Ferner werden die Gleichgewichtskonstanten $K_{p}$ für die Bildung der entsprechenden Verbindungen aus den Elementen im Normalzustand berechnet.

Die Daten zeigen eine sehr hohe Stabilität der Wolframoxihalogenverbindungen und deuten auf eine wesentliche Beteiligung der Wolframoxihalogenide am Reaktionsgeschehen in den Systemen Wolfram - Halogen - Sauerstoff.
\end{abstract}

\section{Problemstellung}

Die chemischen Gleichgewichtszustände der Systeme Wolfram - Halogen und Wolfram - Sauerstoff sind in vorangegangenen Arbeiten ${ }^{1,2}$ vom Standpunkt der Thermodynamik untersucht worden. Dabei wurden aus der Temperaturabhängigkeit des Massenbilanzverhältnisses der jeweiligen Konstituenten der Systeme wichtige Aussagen über die Möglichkeit von chemischen Transportreaktionen und die Richtung dieser Prozesse gewonnen.

In den letzten Jahren ist nun in einer Reihe von Arbeiten ${ }^{3,4}$ die fundamentale Bedeutung des Sauer-

1 G. M. Neumann u. G. Gottschalk, Z. Naturforsch. 26 a, 870 [1971].

2 G. M. Neumann u. G. Gottschalk, Z. Naturforsch. 26 a, 882 [1971]. stoffs für die in Halogenglühlampen ablaufenden Transportreaktionen auf der Basis des Systems Wolfram - Jod erkannt worden, und in jüngster Zeit sind Beobachtungen ${ }^{5}$ gemacht worden, die auf eine Beteiligung des Sauerstoffs auch an den Transportreaktionen im System Wolfram-Brom hinweisen.

Zum Verständnis der Vorgänge in diesen Reaktionssystemen ist die Kenntnis der chemischen Gleichgewichtszustände von großem Interesse. In der vorliegenden Arbeit werden zunächt die für die Gleichgewichtsberechnungen notwendigen thermodynamischen Daten der entsprechenden Wolframoxihalogenide ermittelt und zusammengestellt.

\footnotetext{
3 J. H. Dettingmeijer, J. Tillack u. H. Schäfer, Z. Anorg. Allg. Chem. 369, 161 [1969], sowie dortige Literaturzitate.

4 S. K. Gupta, J. Phys. Chem. 73, 4086 [1969].

5 E. G. Zubler, J. Phys. Chem. 74, 2479 [1970].
} 